\title{
Docencia en tiempos de Covid-19. Aspectos éticos
}

\section{Teaching in times of Covid-19. Ethical aspects}

\author{
PIÑA Elizabeth ${ }^{1}$ \\ PADRÓN-NIEVES Maritza del C. ${ }^{2}$
}

\begin{abstract}
Resumen
La educación es un derecho y un deber social fundamental. Sin embargo, las últimas encuestas colocan en entredicho la calidad de la educación existente, lo cual se agravó con la pandemia por Covid-19. El objetivo de este trabajo fue revisar los aspectos éticos involucrados en la utilización de las tecnologías de la información y comunicación (TIC) en el contexto venezolano y resaltar las recomendaciones nacionales e internacionales para alcanzar una educación de calidad.
\end{abstract}

Palabras claves: ética, docencia, TIC

\begin{abstract}
Education is a fundamental social right and duty. However, the latest surveys question the quality of existing education, which was aggravated by the Covid-19 pandemic. The objective of this work was to review the ethical aspects involved in the use of information and communication technologies (ICT) in the Venezuelan context and to highlight the national and international recommendations to achieve a quality education.

Keywords: ethics, teaching, ICT
\end{abstract}

\section{Introducción}

Una de las definiciones de educación, señala que se refiere al derecho a alcanzar el mayor desarrollo posible de la personalidad, de manera que se pueda disfrutar de la vida personal y social de forma integrada y plena. Es por esto que existe todo un basamento legal que promueve el compromiso del Estado para a adoptar las providencias económicas y técnicas, para lograr la universalidad de este derecho, sin distinciones de edad, sexo, origen social, étnico o cualquier otro status. El cumplimiento de este derecho asegura que sean respetados los principios éticos de beneficencia, autonomía y justicia.

Por otro lado, la globalización de la educación, tiene como herramienta, que favorece la equidad, la incorporación de las redes sociales y el desarrollo tecnológico que permite modalidades de educación a distancia que promueven la comunicación a través del uso multimedia de conocimientos y aprendizajes, lo cual, también conlleva a la revisión de otros aspectos éticos como sonla privacidad, la neutralidad, la brecha digital, el delito cibernético, la transparencia, el plagio, la propiedad intelectual, la responsabilidad, la honestidad, entre otros. De manera que, al ser un sistema de enseñanza-aprendizaje que se desarrolla parcial o totalmente a través de

\footnotetext{
${ }^{1}$ Profesor Titular. Escuela de Enfermería. Facultad de Medicina, UCV. eeeucv@gmail.com. Centro Nacional de Bioética. (CENABI)

2 Profesor Titular. Escuela Luis Razetti. Facultad de Medicina, UCV.mpadron43@gmail.com
} 
las tecnologías de la información y comunicación, requiere de una serie de condiciones para que su implementación sea eficaz y éticamente aceptable.

La realidad del sistema educativo venezolano es que existen debilidades en la infraestructura de las instituciones, además no hay un seguimiento de la calidad de la educación,a lo que se suma la situación económica de estudiantes y docentes que afectan su alimentación, vestido, facilidad de traslado, así como el acceso a servicios de salud. Estas situaciones, se acentuaron con la pandemia por Covid-19 a partir de marzo de este año, cuando se suspendió el proceso escolar presencial para implementar un programa denominado "la escuela en casa", que ha sido considerado por los expertos como improvisado ya que no tomo en cuentalas fallas de conectividad y las deficiencias en los servicios básicos. En este sentido, Bravo Jáuregui (2020) señala además, como una grave lesión al derecho humano a la Educación, la forma como se inicio el año escolar 2020-2021.

En el siguiente trabajo, se realizó una revisión del tema partiendo del basamento legal, describiendo las ventajas y desventajas del uso de las tecnologías de la información y comunicación (TIC), así como la situación actual de la educación en Venezuela, contrastándolas con los aspectos éticos relacionados quetienen una significación moral en el proceso de aprendizaje.

\section{Desarrollo}

\subsection{La educación como derecho}

El derecho a la educación ha sido reconocido expresamente en Declaraciones Internacionales entre la que destaca, la Declaración Universal de los Derechos Humanos (1948) que señala en el artículo 26: “Toda persona tiene derecho a la Educación, la cual debe ser gratuita, al menos en lo concerniente a la instrucción elemental y fundamental...".

Más adelante, en 1952, en el Protocolo Adicional No. 1 al Convenio para la Protección de los Derechos Humanos y las Libertades Fundamentales, se señala en el artículo 2: "A nadie se le puede negar el derecho a la instrucción. El Estado en ejercicio de las funciones que asuma en el campo de la educación y de la enseñanza, respetará el derecho de los padres a asegurar esta educación y esta enseñanza conforme a sus convicciones religiosas y filosóficas".

En Venezuela, este derecho ha evolucionado paulatinamente y la Constitución de la República Bolivariana de Venezuela de 1999, lo incluyó en los Derechos Culturales y Educativos, que lo reconoce como clave para fortalecer las instituciones democráticas, promover el desarrollo del potencial humano, el alivio de la pobreza y fomentar el entendimiento entre los pueblos, ya que debe promover en el Estado, cambios sociales, políticos, económicos, culturales, entre otros. (Olivares García, 2007).

\subsection{Educación, Tecnologías de la Información y Comunicación (TIC) y Aspectos éticos}

Antes de analizar los aspectos éticos es necesario revisar cual es la misión de la educación, al respecto, GómezMartin (2020) señala que es el formar personas en su integridad. Por su parte, Hortal (2000) se remite al informe Delors de 1996, donde se enuncian los objetivos de la educación, sintetizados en la frase: aprender a conocer, aprender a aprender, aprender a vivir juntos y aprender a ser. Hortal, amplía su discurso, al señalar que en las instituciones educativas, además de impartir enseñanzas, también se contribuye a la formación de la persona, a fin de que pueda participar plenamente en la vida y en la cultura de la sociedad en que le corresponde vivir; indicando a los principios bioéticos de beneficencia, autonomía y justicia como su norte moralizador, tres (3) principios prima facie (no absolutos); en donde, el principio de beneficencia se refiere a la obligación moral de actuar en beneficio de otros, promoviendo sus legítimos intereses; el principio de autonomía, se resume en dos condiciones esenciales, la libertad (actuar independientemente de las influencias que pretenden controlar) y ser 
agente (tener la capacidad de actuar intencionadamente); el principio de justicia, se considera como trato igual, equitativo y apropiado, a la luz de lo que se debe a las personas (Beauchamp y Childress, 1999).

Siguiendo el hilo de elementos a considerar ante la definición dada por Gómez-Martin, se tiene que persona es "toda vida humana dotada de capacidad racional y agencia moral, es decir, de responsabilidad y libertad. Es la vida humana con desarrollo de sus cuatro dimensiones: biológica, psicológica, social y espiritual” (Castillo-Valery, 2006 p. 70). La imagen tradicional del docente lo presenta como el profesional que sabe mucho, que guía el proceso educativo, el dador, cuya función es enseñar a quienes poco o nada saben de determinado tema, presentando a los estudiantes como pasivos receptores. Visto de esta manera, el ser educador estaba caracterizado por determinadas habilidades comunicacionales, saberes pedagógicos (metodología de la enseñanza, concepciones del aprendizaje) y disciplinares (conocimiento de la asignatura que imparte), como el criterio de organización y disposición en el aula (dónde se ubica y cómo gestiona el espacio del aula), las actitudes que debe tener como enseñante (temple, escucha, seguridad, paciencia), el modo de vinculación y relación con los estudiantes (apertura, respeto, orientación, cuidado) (Casablancas, 2017). Imagen que Morin (2015) plantea debe ser superada y revitalizada, señalando "enseñar es enseñar a vivir", es decir, aprender a partir de las propias experiencias, asumiendo el error y la ilusión de una decisión, tanto como ciudadanos de la nación, como en su vida personal, guiados primero por los padres y luego por los educadores, con el soporte de los libros y de los encuentros, los cuales implican estar preparados para la confrontación y la comprensión mutua.

Cabe resaltar, que la sociedad actual ha sido impactada por los avances tecnológicos, lo que ha ocasionados grandes transformaciones, particularmente en lo referido a las tecnologías de la información y comunicación (TIC), cambiando la forma y la velocidad de acceso a todo tipo de información, representando un gran reto en el campo educativo, así como abriendo nuevas posibilidades, evidenciadas tanto en la diversificación de las modalidades educativas como en el uso de recursos digitales en el proceso de enseñanza-aprendizaje, cuidando entre otros aspectos, lo referido a la "post-verdad", caracterizada por el peso de las emociones sobre los hechos objetivos y concretos, la información sesgada por intereses de diversa naturaleza o los datos incompletos o falsos, lo que ha derivado en una transformación de los roles del docente y el estudiante. El docente ha de combinar competencias en lo académico, lo tecnológico y la gestión de procesos educativos, además de estimular el trabajo cooperativo y motivar al estudiante; mientras el estudiante, con una participación mas activa, desarrolla la autogestión, el trabajo en equipo y la socialización en ambientes virtuales (Rosas-Chávez, 2017).

La educación a distancia, no es algo nuevo, es definida por García (1996, citado por Pagano 2008), como "un sistema tecnológico de comunicación bidireccional, que puede ser masivo, basado en la acción sistemática y conjunta de recursos didácticos y el apoyo de una organización y tutoría que, separados físicamente de los estudiantes, propician en éstos un aprendizaje independiente", por su parte Martínez-Uribe (2008) lo conceptualiza como el proceso de enseñanza aprendizaje que se da cuando el profesor y el alumno no se encuentran frente a frente como en la educación presencial, empleándose otros medios para la interactividad síncrona (en tiempo real) o asíncrona (en diferido). Inicialmente, se relacionó con la educación de adultos, especialmente a quienes vivían en sitios distantes a los centros educativos, se empleaba la correspondencia u otros recursos como cuadernillos, guías de instrucción, libros, discos compactos (CDs) y programas en la televisión y la radio. En su proceso evolutivo, surgió el concepto de aprendizaje abierto, referido a la libertad de elección del cómo, cuándo y dónde aprender, dando lugar a las universidades abiertas. Con el desarrollo de las TIC, hoy en día se emplean videos, video-conferencias, sesiones de chat y cualquier otro recurso de redes sociales. En la educación a distancia, la mediación tecnológica es de suma importancia, puesto que hace posible la comunicación a través del uso multimedia, lo que permite compartir, conocimientos y aprendizajes; siendo el alumno el centro de las actividades, no el profesor, como en la modalidad presencial, y su fundamento es el autoaprendizaje. 
Con el desarrollo del internet, ha surgido la enseñanza virtual, un nuevo modelo basado en el uso de multimedia interactivo, donde la comunicación es mediada por computadora a través de internet, también se le conoce como de campus virtual o enseñanza virtual, igualmente se puede dar de forma síncrona o asíncrona a través de comunicaciones por audio, video, textos o gráficos, superando la lentitud en la retroalimentación del proceso de aprendizaje de los estudiantes, lo que representaba una de las grandes dificultades de la educación a distancia, ya incluso se pueden presentar pruebas por esta vía y rápidamente recibir la respuesta. (García-Aretio, 2002). Las TIC permiten la interacción con el estudiante en cualquier lugar siempre que se tengan los equipos que lo permitan y la conectividad, sin restricciones del horario escolar y el espacio físico, permitiendo adaptar el ritmo del aprendizaje según sea necesario. (Martínez-Ruiz, 2015).

La tecnología representa una gran oportunidad para salvar problemas derivados del difícil acceso a la información, de dificultades para llegar a centros educativos por cuestión de distancia, entre otros, sin embargo, pretender que en ella recaiga todo el peso de la formación, además de arriesgado, exige de una revisión profunda de las metas propuestas; ya que el incorporar las TIC, reclama estudiar en forma consciente toda la complejidad de esta transformación, lo que representa el peso de la responsabilidad de quienes llevan las riendas de estos procesos. (Mitcham, 2011).

Todo el avance tecnológico, a la par del gran potencial que representa para el acceso a la información de la población, también significa una amenaza para la humanidad. Es aquí donde los seres humanos deben asumir una postura responsable, considerando las posibles consecuencias, evitando caer en la tecnocracia, derivada de la tecnolatría y la enajenación de la sociedad, con especial cuidado a los grupos vulnerables como lo son los niños y jóvenes en proceso de formación. La tecnolatría esclaviza al hombre ante los productos de su propia creatividad. La enajenación, se opone a la emancipación y a la autorrealización del ser humano, a la libertad intelectual y operativa, claro está sin caer en la tecnofobia. Se ha de lograr un equilibrio, buscando la humanización de la técnica al servicio del hombre (Meeroff y Candioti, 1996).

Caccuri (2018) señala las competencias para poder dar un uso adecuado de las TIC: "tener la capacidad para buscar, procesar y comunicar información, transformándola en conocimiento, seleccionando la más relevante, haciendo uso de diferentes soportes, de manera crítica, responsable y segura". Por su parte, Valencia-Molina et al (2016) detallan las habilidades que debe reunir el docente del siglo XXI, derivadas de la transformación de la humanidad en una sociedad de la información y del conocimiento mediada por las TIC: 1. Habilidades psicoeducativas: que le permitan desarrollar capacidades de creatividad para el diseño de experiencias que promuevan el pensamiento crítico, identificación de temas de interés en la disciplina o en el entorno y la evaluación integral de los aprendizajes. 2. Habilidades vocacionales y de liderazgo: que le faculten para la orientación innovadora, creativa y responsable de aquellos que están bajo su guía, aplicando recursos a los que tenga acceso y las metodologías para la enseñanza y la evaluación. 3. Habilidades colaborativas y cooperativas: desarrollando habilidades comunicativas con sus congéneres, compartiendo y creando información y conocimiento logrado a partir de las TIC.

Lezama (2008) plantea realizar una adaptación de la ética como reguladora, acorde con la civilización tecnológica, la cual tiene en este campo, uno de sus más importantes ejes y no un enemigo invencible. Lo que si es cierto, es que la tecnología sigue avanzando a un ritmo que supera la velocidad con que la educación lo ha hecho, lo que la deja en rezago para poder aprovechar el gran campo de oportunidades que se tiene en frente; siendo ya el momento de revisar que se debe enseñar, como y para qué. Las competencias a adquirir durante el proceso de formación siguen siendo las mismas, lo que ha cambiado es el modo como se accede a la información, como se interactúa, lo importante no ha de ser el soporte, sino la capacidades necesarias para la vida; en el contexto digital actual, es necesario repensar y rediseñar el cómo se da el proceso académico, reforzando aquellos aspectos que responden a los nuevos fundamentos del aprendizaje del siglo XXI, el pensamiento crítico y la 
resolución de problemas (Caccuri, 2018). Este autor, indica además que el docente tieneel rol de cuidar que se preserven los derechos fundamentales, estando alerta ante la incursión de elementos que pongan en riesgo la equidad en el acceso y garantizando el buen uso de los recursos y seguridad del usuario.

Olcott et al (2015) al referirse a la incorporación de las TIC en la docencia recomienda hacer una revisión de los aspectos éticos como son la privacidad, la neutralidad, la brecha digital, el delito cibernético y la transparencia, entre otros temas. Un aspecto que destaca, es el referido a la identidad digital, a partir de la cual se va a construir la opinión social o reputación que Rivera-Piraguata y Minelli (2017) la definen como la expresión hiperexistencial del hombre, usuario de la tecnología y que proyecta en el ciberespacio. A ese nivel la identidad estará definida por lo que la persona hace, proyecta y los demás reconocen de él, sin embargo, los autores alertan que no siempre se corresponde con la realidad, pues en ocasiones, intereses egocéntricos pueden intervenir y modificarla, lo que podría estar lesionando aspectos como la responsabilidad y el empoderamiento del individuo, es por ello que durante el proceso de desarrollo y socialización en el campo educativo, este elemento ha de ser considerado; señalando incluso que la responsabilidad es compartida por el docente.

Relacionado con el aspecto identidad, están los temas de plagio y el de propiedad intelectual. Respecto al primero, no siempre es intencional y es practicado con más frecuencia de lo que sería de esperar. Tanto los estudiantes como los docentes reconocen haber incurrido en esta falta u otros tipo de fraude académico. Olcott et al (2015) señalan que de no ser controlado el plagio, podría convertirse en el preludio de un fraude en el ejercicio de la práctica profesional. Más aún, Rivera y Minelli (2017) resaltan la acción docente en procura de controlar este tipo de actuaciones y para ello recomiendan "establecer parámetros y planteamientos pedagógicos que incorporen la visión de que la tecnología, más allá de ser una herramienta, tiene significación moral en el proceso de aprendizaje".(p 55).

Al respecto, Martínez Negrete (2014) reviso varios códigos de conducta que buscaban orientar el quehacer académico enmarcado en valores como la honestidad, el compañerismo y el respeto, encontrando que prácticamente todos se orientaban hacia los códigos de conducta dentro de las instituciones educativas, focalizados en aspectos normativos, dejando de lado lo formativo. En esa búsqueda, encontró el código formulado por Ruth Camuse (2010), el cual fue diseñado bajo el pensamiento de Howard Gardner, expresado en el libro las cinco mentes del futuro (disciplinada, sintética, creativa, respetuosa y ética) donde propone de forma diferencial códigos de ética para estudiantes y docentes. Los aspectos de respeto y ética del estudiante, son: el trato cortes y respetuoso, la prudencia al emitir un comentario, el apoyo a los compañeros, el agradecer y reconocer los aportes de terceros, el ser honesto, el cumplir con las leyes de derecho de autor y buscar la excelencia. En cuanto al código de ética de los docentes indica: el trato cortes y respetuoso, el evitar comentarios despectivos, el promover la comunicación, el respetar la privacidad de los estudiantes, el ser tolerantes con las opiniones, el estar vigilante ante conductas inapropiadas, el actualizar y mejorar constantemente el curso, el escuchar y retroalimentar a los estudiantes, el ser modelo en comportamiento ético, el procurar un sistema de evaluación justo, el esforzarse en reconocer a sus estudiantes para poder dar asesorías personalizadas e igualmente, buscar la excelencia. Culmina Martínez-Negrete (2014) enfatizando la necesidad de códigos de ética orientativos, que promuevan en el estudiante su formación integral, incluyendo aspectos cognitivos, actitudinales y habilidades.

Por otro lado, Cookson (2002) señala que para que las instituciones puedan ser eficaces en su intento de aumentar el acceso y garantizar la equidad, se requiere un compromiso genuino de calidad, para ello presenta cinco criterios que han de aplicarse para la excelencia: el esfuerzo, la actuación, la capacidad, la eficiencia y el proceso. 
El esfuerzo, es quizás una de las variables medidas con mayor frecuencia, tiene que ver con la producción académica, traducida en títulos, inscripciones, exámenes, etc. Sería de esperar que en las modalidades a distancia o abiertas las cifras superaran a las de universidades convencionales.

El segundo criterio, el desempeño, se refiere a los efectos de las actividades de la institución sobre sus funcionarios, para ello es preciso revisar el desempeño de los estudiantes, los logros de aprendizaje y rendimiento en los exámenes, lamentablemente, las investigaciones orientan a que en la modalidad a distancia se da un alto índice de deserción, cuyos factores intervinientes han de ser considerados a la hora de planificar las ofertas, en la búsqueda de mitigar los factores causales.

La capacidad, tercer criterio, se refiere a la habilidad de las instituciones para satisfacer las necesidades educativas y sociales de sus estudiantes, para ello los centros educativos a nivel superior deben evaluar constantemente, que demandan los sectores económicos productivos y de empleo a fin de mantener la vigencia de los programas.

El cuarto criterio, la calidad es el referido a la eficiencia, concerniente al costo; en la educación abierta, suele variar los montos, está el costo por estudiante y el costo por estudiante exitoso; esta relación suele verse afectada numéricamente si se compara con las instituciones de modalidad presencial, la literatura suele explicar que esto ocurre por la posibilidad de seguimiento y apoyo que puede ofrecerse en el sistema presencial, es por ello que una vez admitidos los estudiantes en la modalidad a distancia deben ser guiados a través de sus programas de estudio, lo cual implica, no solo facilitar la tecnología instructiva sino también la generación de apoyos que promuevan su permanencia y culminación exitosa (tutores, cursos de recuperación, etc.), lo cual conlleva al incremento de costos por estudiante, pero aumenta las posibilidades de sobrevivencia en el programa. Específicamente con relación al tutor, Regalado-Espinoza (2011) enfatiza que es quien atiende los requerimientos de su tutelado y garantiza el acceso a la información pertinente, lo ayuda a estructurar el conocimiento, a la vez que se cumplen los acuerdos que ayudaran a reafirmar los rasgos de responsabilidad (tiempos de entrega, pautas para elaborar las asignaciones, búsqueda de apoyos); correspondiendo este actuar al rol beneficente que está llamado a desempeñar el docente, lo que implica ser empático, es decir, ubicarse en la realidad del estudiante.

El quinto y último criterio es el de proceso, referido a las acciones y operaciones que conducen a la meta en forma exitosa y están relacionados con cinco subsistemas, 1. Cursos (creación, producción, distribución y evaluación de la enseñanza). 2. Los estudiantes (todos los procesos de gestión y control del progreso de éstos). 3. Aspectos regulatorios (practicas del personal y unidades académicas y administrativas que toman decisiones con respecto al gobierno, gestión y asignación de recompensar y responsabilidades dentro de la institución). 4. Logística (función de procurar y suplir los recursos requeridos) y 5. Tecnológico (infraestructura de la tecnología de información y comunicación articulada al diseño y entrega de instrucciones).

De manera que, las TIC aunque son una herramienta de avanzada en el proceso educativo, requieren aún de la participación de docentes ó cuidadores formados, para que se cumpla a cabalidad, respetando el derecho a la educación del niño o adolescente y los principios éticos inherentes.

En este orden de ideas, Figueroa (2020) coordinadora nacional de la Unidad Democrática del Sector Educativo señala además, que la educación a distancia, al ser un sistema de enseñanza-aprendizaje que se desarrolla parcial o totalmente a través de las tecnologías de la información y comunicación, requiere de una serie de condiciones para que su implementación sea eficaz, como se ha descrito en esta sección. En el caso venezolano, la educación a distancia pasó a ser un fin en sí misma y no un medio de apoyo para el proceso educativo. 


\subsection{Efectos de la pandemia en la educación}

A finales de 2019, desde Wuhan (China) hacia casi todos los países del mundo se ha expandido una epidemia de enfermedad por coronavirus Covid-19, causada por el virus denominado SARS-CoV-2. Hasta el día 28 de septiembre de 2020, se ha informado de más de 33.5 millones de casos de la enfermedad en 218 países. (Johns Hopkins University, 2020). Desde entonces, se han publicado un gran número de trabajos científicos que intentan describir sus características clínicas, severidad, tratamiento y evolución. Se ha documentado que la transmisión del SARS-CoV-2 ocurre principalmente a través de las partículas respiratorias, de esta manera el virus es liberado en las secreciones de una persona infectada al toser, estornudar o hablar con otro individuo, aun cuando la misma posea únicamente síntomas leves o se encuentre en el periodo pre sintomático (Yang et al, 2020).

La situación mundial es única. Se considera la mayor crisis social en lo que va del siglo XXI, comparable solamente a las mayores catástrofes del siglo XX como las guerras mundiales o el colapso económico de los años 30, por no hablar de las pandemias, especialmente la de 1918-19. Una pandemia como la actual no implica solamente un virus que ataca cuerpos, involucra, además, una alteración del orden social, económico, político y psicológico (Lolas Stepke, 2020). De manera que estamos ante nueva categoría que reúne lo social y lo biológico, por eso algunos autores lo han denominado como sindemia, que es un término que hace referencia a problemas de salud de una población en sinergia con sus contextos sociales y económicos.

A nivel mundial, casi la totalidad de instituciones educativas de todos los niveles debieron cancelar sus actividades presenciales, en cumplimiento de las disposiciones adoptadas por las autoridades de los distintos países, tendientes a disminuir la propagación de la pandemia y sus consecuencias fatales. Según un informe de UNICEF, a inicios de mayo de 2020 aproximadamente 1.287 millones (90\% del total de estudiantes del mundo), estaban afectados debido al cierre de instituciones educativas, 182 países continuaban la interrupción total de los servicios de educación y 8 lo hacían parcialmente de modo local en distintas jurisdicciones. (UNICEF, 2020)

Ahora bien, una de las consecuencias del cierre de escuelas en respuesta a Covid-19 ha producido que se evidencien diversos problemas socioeconómicos, tales como: falta de formación para elaprendizaje digital, tanto de los docentes como de los estudiantes, el poco acceso a internet, la seguridad alimentaria, los deficientes servicios de salud y la falta de servicios destinados a personas con alguna discapacidad. (UNESCO, 2020).

A través del Laboratorio Latinoamericano de Evaluación de la Calidad de la Educación (LLECE) (2020), promovido por la UNESCO, se revisaron las condiciones de los sistemas educativos de la América Latina, encontrando que las diferencias socioeconómicas debían ser consideradas, pues eran los alumnos provenientes de niveles más bajos, los que presentaron mayores dificultades para responder a los cambios incorporados. El $100 \%$ de los países estudiados (18 países) declararon faltas en la infraestructura necesaria para la conectividad (computador e internet), a ello se le agrega la brecha digital entre las zonas urbanas y rurales, cuyo promedio supero los 10 puntos porcentuales en las zonas mas remotas, las dificultades se extiende a la falta de electricidad o de transporte, lo cual dificulta incluso la entrega de material físico; otro de los elementos estudiados fueron las competencias TIC, encontrando niveles bajos en toda la región, así como escasa presencia en las mallas de formación docente y en los currículos escolares (11 países); en lo correspondiente a los materiales disponibles, en 4 países fue clara la falta de calidad, no adaptados a los currículos escolares bajo la nueva modalidad; en cuanto a la búsqueda de alternativas para mantener el vínculo docentes-estudiantes y sus familias, se había acudido a la mensajería instantánea. Dado los resultados del estudio, la organización recomendó la revisión y perfeccionamiento de las metodologías y estrategias empleadas, sugiriendo además, el uso de modalidades híbridas, así como el diseño de estrategias para disminuir las debilidades encontradas, con especial interés en los estudiantes marginados. 
López-Calva, enfatiza que la desigualdad educativa existente en América Latina puede incrementarse exponencialmente debido a que a los problemas antes mencionados, se suman las diferencias del nivel educativo alcanzado por los padres o cuidadores, responsables en esta instancia de apoyar el proceso de aprendizaje de los niños (López-Calva, 2020).

Con relación a la aparición de primeros casos de Covid-19 en Venezuela, fueron reportados el 13 de marzo de 2020 y 8 días después se informó de la primera muerte. Sin embargo, se supo después, de un caso que presentaba síntomas desde el 29 de febrero de este año bisiesto, por ello, el Gobierno declaró estado de alerta y una cuarentena obligatoria por 30 días a partir del 17 de marzo y que continúa a la fecha, ocho meses después.

El contagio de la enfermedad, depende del número de personas que se infectan, de la transmisibilidad de la infección y de su espectro clínico, así como de la severidad. Es por esto, que surgió la necesidad del aislamiento social con el consecuente impacto, no solo a nivel sanitario y la salud mental, sino también en los aspectos socioeconómicos, el mercado de valores, el financiero, la aviación, el transporte y la educación. (Lipsitch et al, 2020). Cabe acotar, que en Venezuela, buena parte de las restricciones que aún se mantienen, son el resultado de la combinación de: medidas derivadas del Covid-19 yla crisis de abastecimiento de gasolina. Hasta $43 \%$ de los hogares venezolanos señalaron en la Encuesta de Condiciones de Vida (Encovi) imposibilidad de trabajar o incluso, la pérdida de sus ingresos. (Encovi, 2020).

\subsection{La Educación en Venezuela}

La calidad de la educación en Venezuela estaba bajo la lupa, ya que al ser evaluada, mostró una tendencia negativa en comparación con años anteriores. El análisis de la situación demostró que era necesario debatir temas como: mejorar sustancialmente la educación pública, incrementar el número de planteles en todos los niveles desde básica a superior, de preferencia con comedores para los estudiantes, incrementar los beneficios económicos al profesorado, incentivar la investigación, elevar los índices académicos para optar a la carrera de educación, incrementar la oferta de especialización y maestrías al profesorado, revisión de los pensa académicos e incorporación de materias fundamentales que permitan la comprensión del mundo actual. (Yeliann-Cano, 2016).

En este sentido, algunos datos que aporta Encovi 2019 - 2020, son el registro de una caída en la demanda potencial de la educación. Entre 2014 y 2018, la demanda era de 12.700 .000 pero cayó a 11.000 .000 estudiantes. Solo $60 \%$ de la población escolarizada es la que consigue asistir a clases y casi 4 millones de niños están en situación de vulnerabilidad, principalmente, por la falta del suministro de comida, tanto en el plantel como en el hogar, fallas de servicios básicos en las escuelas y la falta de medios de transporte. Las escuelas privadas han tenido que incrementar exponencialmente el costo de sus mensualidades para pagar más a sus docentes y mantener la calidad de su enseñanza y no tienen garantía en su derecho a la educación.

Las demandas derivadas de la aparición de la pandemia por Covid-19 y el llamado a imponer distancia física entre las personas fue asumido en Venezuela, con un Programa Educativo del Ministerio del Poder Popular para la Educación Ilamado "Cada familia una escuela" supuestamente inspirado en la educación a distancia, donde se proponía realizar diariamente algunos encuentros virtuales y proporcionando una serie de orientaciones que los docentes elaboraron de forma apresurada, surgiendo una serie de interrogantes, respecto a que garantías existen para poder responder a los criterios formulados anteriormente respecto a los objetivos de la educación.

La suspensión de actividades presenciales en las escuelas llevo a la declaración de culminación del año escolar, lo cual para el Observatorio Educativo de Venezuela (OEV) fue extremadamente accidentado y muy mal planificado ya que, sin haber realizado las evaluaciones pertinentes, no se puede hacer un símil entre educación a distancia y la educación en casa, por más que la modalidad a distancia se desarrolle en el hogar de cada estudiante. 
En este sentido, Ramos (2020) señala que para iniciar un nuevo período escolar en esta situación de emergencia, se deben considerar: a) medidas extraordinarias con planificación y sustentación técnica. En educación no se puede improvisar; b) desarrollar educación a distancia que garantice formación de calidad; c) considerar que no toda la población estudiantil podrá incorporarse a este sistema con base en las desigualdades socio-territoriales que se incrementaron durante la pandemia; d) debería contarse con un sistema de acompañamiento y supervisión por una red de mensajeros del Ministerio de Educación; e) elaborar los programas de estudio con una evaluación adecuada que permita verificar los aprendizajes desarrollados porque es considerable el impacto que una educación incompleta y de baja calidad tiene en la vida de las personas y de cómo eso afecta, no sólo la vida de quienes no tienen acceso a educación de calidad, sino también su propia calidad de vida.

Por otra parte, las instituciones de educación superior autónoma, públicas y privadas, no escapan a esta situación: su matrícula es cada vez menor y hay una elevada deserción de docentes. El Gobierno las tiene sometidas a un cerco presupuestario en procura de su cierre y, por el contrario, ha creado numerosas universidades improvisadas. Según la Encovi 2019-2020, se registraron 3.136 .000 estudiantes (entre 18 y 24 años de edad) y encontraron un retroceso aún mayor en la cobertura educativa. Ya que del 2014 y al 2019 se redujo a la mitad. De estos, solo 775.000 asisten a clases y solo 155.000 se gradúan. Con la pandemia, este panorama se agudizó. Como ejemplo, en la Universidad Central de Venezuela, la Asociación de Profesores de la UCV (APUCV)(2020), realizó una consulta a 1.624 docentes, la mayoría activos (81,1\%), en la que encontraron que las condiciones tecnológicas para poder iniciar actividades, están comprometidas ya que el $97 \%$ de éstos, financia el servicio de internet, el cual no es constante $(64,9 \%)$ y solo el $21,4 \%$ lo calificó de bueno o muy bueno. De manera que, la calidad de la conectividad dificulta el manteniendo del distanciamiento físico y que los docentes puedan interactuar con los estudiantes desde su casa.

Otro estudio que presenta la situación del sistema educativo venezolano en los momentos de pandemia, fue el publicado por la Unidad Democrática del Sector Educativo (UDSE) (2020), quienes encontraron que los sectores sociales mas pobres son los afectados en mayor medida, por su dificultad para responder a la modalidad implementada, es ahí donde no se cuenta con acceso a la tecnología; en su informe presentan los datos del Observatorio Venezolano de Servicios Públicos (OVSP), quienes a su vez encontraron que para el 2019, solo el $40 \%$ de los hogares venezolanos tenían acceso a internet, ubicando a los sectores sociales $D$ y $E$, como los menos favorecidos, por ser el grupo poblacional sin el servicio. Igualmente, en el informe de la UDSE (2020) se destaca el comportamiento dispar entre escuelas públicas y privadas, estando en estas últimas, la población con menos dificultades para cumplir con el llamado a la "educación a distancia"

Rivera y Minelli (2017) alertan sobre el riesgo a que están expuestos determinados grupos sociales, de no poder permanecer en el sistema educativo en forma exitosa, cuando se implementan programas de educación a distancia, por no contar con posibilidades de conectividad, siendo esto una inequidad. Sobre este aspecto, el estudio de UDSE (2020) reportó una disminución de la matricula cuando se implementó la modalidad a distancia, como referencia se señaló lo ocurrido en un sector del estado Miranda, donde un docente indicó que de 23 alumnos inscritos, solo se pudo atender a 10, a pesar de haber diseñado "guías" para aquellos niños que no tenían internet, pero no se logró la respuesta necesaria por parte de los padres; lo cual fue aún más crítico en los casos de educación especial.

Pasar de una modalidad a otra, requiere de una serie de ajustes, a la hora de planificar la incorporación de la educación a distancia, se debe considerar el tiempo que utilizará el docente en la elaboración de materiales, la revisión de trabajos, las asesorías y el apoyo al estudiante, seguimiento y retroalimentación oportuna, lo cual ha de traducirse, no solo en la incorporación de la tecnología u otros recursos, sino también en la remuneración del docente, en caso contrario, se corre el riesgo de asumir posturas facilistas a la hora de evaluar trabajos o cuando se requiere de estimular la reflexión durante los debates, foros, informes de prácticas o pasantías, lo que podría 
propiciar que se pasen por alto conductas deshonestas, como el fraude, corrupción o plagio (Regalado-Espinoza, 2011); así también, se ha de evaluar la capacitación del docente para adaptarse a las exigencias de la nueva modalidad. En Venezuela, ante el éxodo de profesores, en algunas regiones se implementó la política del "docente exprés", improvisando con la contratación de personas sin la debida formación, lo cual representa una grave amenaza para la calidad de la enseñanza. (UDSE, 2020).

El tema de la evaluación requiere especial cuidado, como se ha señalado en los párrafos anteriores la importancia del componente formativo dentro de ella, así como su proyección como indicador de equidad, es por ello, que debe ser abordada en forma consciente y responsable; sin embargo el estudio de LLECE (2020), encontró que la mayoría de los países no tiene claridad de cómo será abordado este aspecto, pareciera que la promoción de estudiantes sea la opción asumida, otros plantean revisar el desempeño durante el año y una evaluación final flexible; esta indefinición podría estar albergando una forma de inequidad, pues se estaría perdiendo la posibilidad de garantizar el nivel de formación buscado.

Como se mencionó en la primera parte, tanto en la Declaración Universal de los Derechos Humanos (1948) como en la Constitución de la República Bolivariana de Venezuela (1999), el Estado está obligado a promover una educación de calidad y universal, para lo cual es necesario tratar de revertir el cuadro de emergencia humanitaria compleja en que se haya sumergido el país; por su parte, la educación es un bien público, que ha de cubrir a la totalidad de la población, la cual ha de ser valorada, respetada, promoviendo su formación como ciudadano, es ahí donde radica la democratización educativa, evitando todo tipo de exclusión. (Regalado, 2011).

Venezuela es uno de los países suscriptores de la Agenda 2030 para el desarrollo sostenible, la cual fue asumida por muchos países en septiembre de 2015. De los 17 objetivos que la conforman, el № 4, está dedicado a la educación de calidad; siendo el nivel educativo de una población el que permite su movilización ascendente en la escala socioeconómica, elemento clave para salir de la pobreza, sin embargo, todavía existe una gran deuda con ciertos grupos poblacionales, sobre todo en el hemisferio sur del planeta. (ONU, 2018). En lo correspondiente a la calidad del sistema educativo, LLECE (2020) señala a los gobiernos como los responsables de promover las condiciones necesarias, correspondiendo al sistema de educación repensar los modelos a desarrollar, sin perder de vista el avance hacia currículos relevantes y pertinentes para los ciudadanos del siglo XXI, conformando sistemas educativos incluyentes y flexibles que den a cada estudiante la posibilidad de desarrollarse según sus fortalezas e intereses. Por otro lado, la UNESCO (2016) afirma que es el Estado el que puede evitar las diferencias entre la gente, trabajando por la disminución de la brecha digital, valiéndose de las comunicaciones satelitales para llegar a las zonas más remotas y desprotegidas; si la cobertura total no es posible, las TIC deben combinarse con tecnologías más tradicionales, como materiales impresos y programas en la televisión y la radio, para lograr más efectividad.

En este mismo orden de ideas, La UDSE (2020) invoca la responsabilidad del Estado, tal como lo señalan los artículos 102 y 103 de la Constitución de la República Bolivariana de Venezuela, lo que implica asumir los costos de las instalaciones de sistemas centrales de transmisión de datos inalámbrica utilizada para Internet (WIFI, de sus siglas en inglés 'Wireless Fidelity', es decir, 'fidelidad inalámbrica')a través de la Compañía Anónima Nacional de Teléfonos de Venezuela (CANTV) a lo extenso del país, de esta manera garantizar a los estudiantes de todos los estratos socioeconómicos la interacción en el proceso educativo a distancia, así como crear una biblioteca virtual que facilite a los estudiantes el encuentro con el conocimiento.

Con la aparición del Covid-19 en el 2020, se inició un progresivo cierre del mundo educativo, llegando a afectar a más del $91 \%$ de los estudiantes a nivel mundial. Esta situación llevo a integrar esfuerzos para fomentar la colaboración internacional, lo que motivo a la UNESCO a promover la Coalición Mundial para la Educación Covid19, que representa una alianza multisectorial entre el sistema de Naciones Unidas, organizaciones de la sociedad civil, medios de comunicación y los asociados de tecnología de la información para diseñar e implantar soluciones 
innovadoras en procura de abordar los vacíos de comunicación y de conectividad, facilitando las oportunidades de aprendizaje inclusivo, promoviendo soluciones equitativas y el acceso universal, siendo gobiernos de los países, a los que les correspondería promover y gestionar localmente la aplicación de las medidas que permitan alcanzar las metas propuestas. (ONU, 2020)

Manteniendo esta línea de pensamiento la Comisión Económica para América Latina y el Caribe (CEPAL) (2020) instó a los países miembros a garantizar y universalizar la conectividad y asequibilidad a las tecnologías digitales para enfrentar los impactos provocados por la pandemia del coronavirus en la región. Para ello, propuso cinco líneas de acción orientadas a construir una sociedad digital inclusiva, para asegurar un 'Estado de bienestar digital'. De manera operativa la propuesta incluye garantizar a cada hogar no conectado una canasta básica de TIC conformada por un computador portátil, un teléfono inteligente, una tableta y un plan de conexión, con un costo anual inferior al 1\% del PIB. Acorde con este planteamiento, en Venezuela, la UDSE (2020) propuso: 1. Recuperar el programa de entrega de computadoras para los estudiantes a fin de que puedan integrarse en la modalidad de educación a distancia y 2 . Diseñar programas de preparación tecnológica y pedagógica dirigida a los docentes para dar las herramientas necesarias sobre la educación a distancia.

Finalmente, para llevar adelante estas propuestas se requiere de la voluntad política por parte del Estado, en caso contrario, las condiciones ya lamentables se agudizarán y solo tendrán acceso a una educación de calidad, aquellos que puedan financiarla.

\section{Conclusiones}

La situación educativa venezolana es realmente compleja, tal como los demuestran los estudios, y la situación de sindemialamentablemente ha agudizado los problemas que se venían arrastrando y se manifiesta como vulneración de los principios antes descritos.

Hoy en día, con el llamado "Cada familia una escuela", se han incrementado las inequidades, pues la convocatoria se realizó sin antes garantizar las condiciones para desarrollar ese plan, derivadas de la carencia en gran número de hogares de la tecnología mínima necesaria, a esto se le suma las fallas constantes de los servicios básicos, como la electricidad. El último trimestre del periodo académico 2019-2020 se caracterizó por la improvisación, hecho que se repite sin grandes variaciones en el inicio del periodo académico 2020-2021. Por otro lado, con los docentes recibiendo sueldos de hambre, se incrementa la huida a otros campos de trabajo, lo que ha derivado en la generación de áreas del saber totalmente desprovisto del talento humano; es la combinación de estos aspectos lo que determina el incremento de la exclusión social. La manera de evitar las brechas sociales radica en cuidar la pertinencia y adecuación del sistema educativo, garantizando el acceso de todos a una educación de calidad, siendo esto responsabilidad del Estado.

\section{Referencias bibliográficas}

Asociación de Profesores de la Universidad Central de Venezuela (APUCV). (2020). Profesores UCEVISTAS expresaron que no hay condiciones para el reinicio de clases bajo ninguna modalidad. APUCV Informa № 1121, 8-10-2020. Caracas. Consulta: 10-10-2020, disponible en: https://app.dc1.emailchef.com/viewonline/@f*025j2f7ib38j2f2S1*3=0183f5f6e6b2f,b*6e3r8ffs2dba9f3s 9dffO*1=92f801532772b8802-2.

Beauchamp, T. y Childress, J. (1999) Principios de Bioética. Barcelona: MASSON, S.A. (p.522.)

Bravo Jáuregui, L. Memoria Educativa 813 del 18 de septiembre de 2020. Consulta: 28-09-2020, disponible en: http://luisbravoj.blogspot.com/. 
Caccuri, V. (2018). Competencias digitales para la educación del siglo XXI [e-book]. Consulta: 09-10-2020, disponible en: https://virginiacaccuri.blogspot.com/2018/06/ebook-gratis-competencias-digitales.html.

Casablancas, S. (2017). No es Malo Perder el Rumbo: Reconfiguraciones del rol docente: En el contexto digital. En: Héctor Sevilla, F. T. y Marisol,L. (coordinadores) Educar en la era digital Docencia, tecnología y aprendizaje. México: Editorial Pandora, S.A. de C.V.

Castillo-Valery, A. (2006). Fundamentación. En: Castillo-Valery, A. y Padrón, M. (editores) Bioética. Caracas: Disinlimed, C.A. (p. 51-88.)

Comisión Económica para América Latina y el Caribe (CEPAL). (2020) Universalizar el acceso a las tecnologías digitales para enfrentar los efectos del COVID-19. Informe especial COVID-19 No 7. Consulta 13-10-2020, disponible en: https://www.cepal.org/es/comunicados/cepal-propone-garantizar-universalizar-laconectividad-asequibilidad-tecnologias

Constitución de la República Bolivariana de Venezuela. (1999). Gaceta Oficial de la República Bolivariana de Venezuela № 36.860. 30 de diciembre de 1999.

Convenio para la Protección de los Derechos Humanos y las Libertades Fundamentales. (1952). Gaceta oficial extraordinaria no. 19.255 del 20 de mayo de 1952.

Cookson, P.S. (2002). Acceso y equidad en la educación a distancia: Investigación, desarrollo y criterios de calidad. REDIE [online]. (4(2):148-167.)

Encuesta sobre Condiciones de Vida en Venezuela-ENCOVI. (2020). Resultados preliminares, 2019-2020, Consulta: 28-09-2020, disponible en: https://retopaisvenezuela.org/encovi-2019-2020-venezuela-es-elpais-mas-pobre-de-latinoamerica/.

Figueroa, R. (2020). "La educación a distancia pasó a ser un fin en sí misma y no un medio de apoyo". Unidad Democrática del Sector Educativo. Artículo de Indira Rojas. Entrevista desafíos de la educación en pandemia. Prodavinci.com.

García-Aretio, L. (2002). Perspectiva histórica. En:La educación a distancia: de la teoría a la práctica. Barcelona: Editorial Ariel S.A.

Gómez-Martin, E (2020). Educación en el Mundo Incierto. Consulta: 26-09-2020, disponible en: https://ethic.es/2020/09/formacion-mundo-incierto/.

Hortal, A. (2000). Docencia. En: Cortina, A. y Conill, J. (coordinadores) Palabras Claves en Ética de las profesiones. España: Editorial Verbo Divino. (p. 56-78.)

Johns Hopkins University (2020). Coronavirus COVID-19 Global Cases. Consulta: 28-09-2020, disponible en: https://www.google.com/search?q=coronavirus+Covid19+global+cases+by+johns+hopkins+csse\&rlz=1C1AOHY_esVE814VE814\&oq=Coronavirus+COVID19+Global+Cases\&aqs=chrome.1.69i57j0l5.3830j0j7\&sourceid=chrome\&ie=UTF-8.

Lezama, J. (2008). Tecnología, naturaleza humana y ética. En: Urdaneta, Alberto; Farías, Haydee y Carmona Miriam (editores) La dimensión ética de las ciencias y la tecnología. Caracas: Editorial Melvin C.A. (p. 111119.)

Lipsitch, M., Swerdlow, D.L., Finelli, L. (2020). Defining the Epidemiology of Covid-19 Studies Needed.N Engl J Med. 382:1194-119. DOI: 10.1056/NEJMp2002125 
LLECE- Laboratorio Latinoamericano de Evaluación de la Calidad de la Educación. (2020). Sistemas educativos de América Latina en respuesta a la Covid-19: Continuidad educativa y evaluación Organización de las Naciones Unidas para la Educación, la Ciencia y la Cultura (UNESCO) julio 2020, Consulta: 05-10-2020, disponible en: https://unesdoc.unesco.org/ark:/48223/pf0000373761.

Lolas Stepke, F. (2020). Editorial Perspectivas Bioéticas en un mundo en sindemia. Acta bioethica 26 (1): 7-8

López-Calva, L.F.(2020). Heyteachers, (don't) leavethekidsalone: Conectividad y desigualdades educativas en tiempos de COVID-19. Programa de las Naciones Unidas para el Desarrollo. PNUD en América Latina y el Caribe.

Martínez-Negrete, E. (2014). Hacia un código de ética para la educación virtual, basado en las cinco mentes del futuro. Revista Mexicana de Bachillerato a Distancia, (11), año 6, febrero de 2014. Consulta: 15-08-2020, disponible en: http://www.revistas.unam.mx/index.php/rmbd/article/view/65017.

Martínez-Ruiz, X. (2015). Educación Virtual: Consideraciones éticas y semánticas desde la infoesfera. Innovación educativa (México, DF), 15(68), 9-14. Consulta: 14-09-2020, disponible en: http://www.scielo.org.mx/scielo.php?script=sci_arttext\&pid=S1665$26732015000200002 \& \operatorname{lng}=e s \&$ tlng=es.

Martínez-Uribe, C. H. (2008). La educación a distancia: sus características y necesidad en la educación actual. Educación (XVII (33) p. 7-27). Consulta: 02-10-2020, disponible en: Dialnet-LaEducacionADistancia5057022.pdf.

Meeroff, M. y Candioti, A. (1996). Ciencia, tecnología y ética. En: Ciencia, técnica y humanismo: Una propuesta a la sociedad. Buenos Aires: Editorial Biblos (p. 65-76.)

Mitcham, C. (2011). La Tecnología y el peso de la responsabilidad. En: Valores y ética para el siglo XXI. BBVA. España: TF editores. Consulta: 10-09-2020, disponible en: https://www.bbvaopenmind.com/libros/valores-y-etica-para-el-siglo-xxi/.

Morin, E. (2015). Enseñar a vivir. Manifiesto para cambiar la educación. Buenos Aires: Nueva Visión.

Olivares García, S. (2007). El derecho a la educación como un derecho humano fundamental a la luz de la Constitución de 1999. Frónesis, (14(2) p. 11-36.) Consulta: 28-09-2020, disponible en: http://ve.scielo.org/scielo.php?script=sci_arttext\&pid=S1315-62682007000200002\&lng=es\&tlng=es.

Olcott Jr. D., Carrera-Farran, X., Gallardo-Echenique, E. y González-Martínez, J. (2015). Ética y educación en la era digital: perspectivas globales y estrategias para la transformación local en Cataluña. RUSC.Universities and Knowledge Society Journal, (12(2) p.59-72.)Consulta: 15-08-2020, disponibleen: http://dx.doi.org/10.7238/rusc.v12i2.2455.

Organización de las Naciones Unidas (ONU). (1948). Declaración Universal de los Derechos Humanos.

Organización de Naciones Unidas (ONU). (2020). Educación de Calidad. Desarrollo Sostenible. Consulta: 13-102020, disponible en: https://www.un.org/sustainabledevelopment/es/education/.

Pagano, C.M. (2008). Los tutores en la educación a distancia: Un aporte teórico. Revista de Universidad y Sociedad del Conocimiento. Consulta: 01-10-2020, disponible en: http://rusc.uoc.edu/rusc/ca/index.php/rusc/article/download/v4n2-pagano/304-1220-2-PB.pdf 
Programa de Naciones Unidas para el Desarrollo (PNUD). (2018). Objetivos de Desarrollo Sostenible. Consulta: 27-02-2019. Disponible en: http://www.undp.org/content/undp/es/home/sustainable-developmentgoals.html.

Ramos O. (2020). En educación no se puede improvisar. Artículo de Indira Rojas. Entrevista desafíos de la educación en pandemia. Prodavinci.com.

Regalado-Espinoza, E.L. (2011). Educación a distancia y calidad ética. EspamCiencia (2(2):37-41. 2011.) Consulta: 15-08-2020, disponible en: file://J:/USB/PCI/Asovac\%202020/Mexico\%20Codigo.pdf.

Rivera-Piraguata, J. y Minelli, J. (2017) El problema ético de la identidad digital en la educación virtual. RevlberoamerEduc. 75(2): 41-58.

Rosas-Chávez, P. (2017). Tecnologías para el aprendizaje y desafíos curriculares. En: Héctor Sevilla, F. T. y Marisol L. (coordinadores) Educar en la era digital docencia, tecnología y aprendizaje. México: Editorial Pandora, S.A. de C.V. (p. 93.)

UNESCO- Organización de las Naciones Unidas para la Educación, la Ciencia y la Cultura. (2020). La educación en América Latina y el Caribe ante la COVID-19. Consulta: 28-09-2020, disponible en: https://es.unesco.org/fieldoffice/santiago/Covid-19-education-alc/respuestas.

UNESCO- Organización de las Naciones Unidas para la Educación, la Ciencia y la Cultura. (2016). la integración de las tecnologías de la información y la comunicación en los sistemas educativos. Consulta: 13-10-2020, disponibleen: https://unesdoc.unesco.org/ark:/48223/pf0000150785.

UNICEF - United Nations International Children's Emergency Fundation.(2020). COVID-19: Preparación y respuesta educative. Respuesta de UNICEF a los desafíos de educación en América Latina y el Caribe durante el COVID-19. Consulta: 28-09-2020, disponible en: https://www.unicef.org/lac/la-educacionfrente-al-Covid-19.

Unidad Democrática del Sector Educativo (UDSE). (2020). Destrucción de la educación como derecho humano. Educación Bolivariana: una gigantesca estafa histórica. Informe 2019-2020. Magisterio Alternativo. Venezuela, julio 2020. Consulta: 10-10-2020, disponible en: http://moveducsimonrodriguez.blogspot.com/2020/07/desde2018-la-unidad-democratica-del.html.

Valencia-Molina, T., Serna-Collazos, A., Ochoa-Angrino, S., Caicedo-Tamayo, A., Montes-González, J., ChávezVescance, J. (2016). Competencias y estándares TIC desde la dimensión pedagógica: Una perspectiva desde los niveles de apropiación de las TIC en la práctica educativa docente. Pontificia Universidad Javeriana - Cali (p.77.)

Yang Y, Pen F, Guan K. (2020). The deadly coronaviruses: The 2003 SARS pandemic and the 2020 novel coronavirus epidemic in China [Internet]. J Autoimmunity. 2020. Consulta: 28-09-2020, disponible en: https://www.sciencedirect.com/science/article/pii/S0896841120300470

Yeliann-Cano, Y. (2016). La situación actual de la Educación en Venezuela. Consulta: 28-09-2020, disponible en: https://sites.google.com/a/correo.unimet.edu.ve/g11617-1s21educacioncsbajas/home/la-situacionactual-de-la-educacion-en-venezuela.

Esta obra está bajo una Licencia Creative Commons Attribución-NoCommercial 4.0 International

(cc) EY-NG 